\title{
Big Data Analytics and Organizational Performance: A Meta-Analysis Study
}

\author{
Mihai BOGDAN ${ }^{1, *}$ \\ Anca BORZA²
}

\begin{abstract}
Big data analytics gained a great deal of attention from both, the business world and the academia. Investments of time, capital and personnel are significant, to the level that pioneers of this field reached a point of no return. Although there are clear insights of successful companies built on a big data analytics strategy, the results from the researchers' work show mixed outcomes. It is not explicit under which circumstances big data analytics adoption leads to positive results. The purpose of this study is to run a meta-analysis review on the empirical articles that tackle big data analytics and organizational performance topics. As a methodology, we searched all the articles indexed on ISI Web of Knowledge during period January 2010 - May 2019. After the first filter, we selected 375, out of which we included only those studies which respected our full criteria: empirical study, provided results, at least one effect size and measurement error displayed. Based on this standard, we included 37 articles in our meta-analysis. The findings confirm that big data analytics play an important role in organizational performance. Finally, implications for practice are discussed, and future research directions are proposed considering the limitations of our study.
\end{abstract}

KEYWORDS: big data analytics, organizational performance, meta-analysis

JEL CLASSIFICATION: $M 15, O 32$

\section{INTRODUCTION}

"Data is the new oil", this is a very common headline from many business newspapers (e.g. The Economist, Medium, Hackermoon etc.). This metaphorically association seems quite right if we think that it highlights many of the similarities between these two, otherwise totally different elements. Firstly, it sends to the idea of the huge economic importance as a resource which both elements possess. Then, like oil, data needs to be "mined" and refined in order to obtain a valuable finished product. Also, the idea that data can be sold as a commodity, so it is not mandatory that the ones that produce it should use it. Even those who wrote articles arguing why "data is not the new oil" somehow just reinforce the idea. If data is going to be a critical value in the future, then the next question is to what extend is big data analytics valuable for an organization. Similar with the emergence of internet in the 90s where many researchers rushed in to argue that there is a clear connection between investments in IT infrastructure and organizational performance, there are many specialists that already drew the conclusion that investing into big data analytics is the right path for all organizations (Gupta \& George, 2016).

\footnotetext{
${ }^{1}$ Babeș-Bolyai University Cluj-Napoca, Romania, mihai.bogdan@econ.ubbcluj.ro, *Corresponding author

${ }^{2}$ Babeș-Bolyai University Cluj-Napoca, Romania, anca.borza@econ.ubbcluj.ro
} 
However, if we are to review the results of the published articles, we might be tempted to deduce that the results are mixed and that there is no decisive conclusion. It is because of this, that the purpose for the current study is to do a meta-analysis review across all the empirical studies on the topic of big data analytics and firm performance.

\section{LITERATURE REVIEW}

One of the first findings we noticed when we started the meta-analysis is that the researchers define, or better put, identify big data analytics in many forms and shapes. For example, some authors classify big data analytics based on the types of analyses used such as: descriptive, predictive and prescriptive analytics (Aydiner et al., 2019). Others make distinction between big data technological capabilities and big data personnel capabilities (Anwar et al., 2018). A third categorization is based on the distinction between big data resources and big data capabilities (Gupta \& George, 2016).We have a multitude of approaches for the measurement of performance. The most common approach encountered is to assess the financial performance or the market performance (Corte-Real et al., 2017; Huang et al., 2018; Ren et al., 2016; Tang et al., 2018; Wamba et al., 2017; Yu et al., 2018). Another cluster used for assessing an organization's effectiveness is the ability to obtain a competitive advantage (Anwar et al., 2018; Shan et al., 2018), others measure the decision-making effectiveness (Wang \&Byrd, 2017).

Regardless of the methods or scales through which the two elements are assessed, our scope and direction is the same, and most simply put, we aim to evaluate the extent to which big data analytics produces positive outcomes. Although this might be considered as too broad or too generalized, we argue that our approach is backed by one of the pioneers of meta-analysis methodology. When being asked about the danger of operating a too inclusive selection procedure, Glass (1978) used the analogy of comparing apples and oranges, saying that it is acceptable to compare the two, if your object of study is the fruit. As a reinforcement of Glass's claim, Rosenthal (1991) continued that combining apples and oranges is desirable if you want a fruit salad. From a statistical point of view, there is no restriction as which studies to be included. However, the question has to be asked as how diverse should be the pool of studies in order for the analysis to be meaningful. It is considered as a good practice, if the question asked by the meta-analysis study is broader than the one asked by the individual studies (Borenstein et al., 2009). Due to the diversity of the studies, it is recommended that the analysis should use the random effects model, but about this topic we will be more comprehensive in the methodology section of this study. We aim to split the literature review within two parts, which is similar to the logic of our study: first, big data analytics within literature and second, measuring approaches for performance within the included articles in our meta-analysis.

\subsection{Big data analytics in the literature}

Big data is defined within the practitioner's world and it seems that these definitions are incorporated in the academic literature as well. According to Gartner Inc: "Big data is highvolume, high-velocity and/or high-variety information assets that demand cost-effective, innovative forms of information processing that enable enhanced insight, decision making, and process automation (Gartner, n.d.). "The focus here is on the characteristics of big data, which are: high in volume, increased velocity and broad variety. The authors acknowledge that data represents an asset, based on which, economic value is pursued. In order to be processed, state-of-the-art tools and specialized knowledge are required. Third, the benefits of putting in place such a process is new discoveries, improved decision-making system, and 
automation. Basically, the validity of the last part is the scope of our study: to assess whether or not this proposition is proven by the results of the articles from the academic world.

Davenport and Harris (2007) describe big data analytics as:"the extensive use of data, statistical and quantitative analysis, explanatory and predictive models, and fact-based management to drive decisions and actions". In other words, the raw material here is data, which is processed using a multitude of statistical methods, and what is happening at organizational level is that a new style of management is put in place, which is based on evidence, contrary to the so called "gut feeling" decisions or managing by one's experience.

Based on the scope, big data analytics, can be classified as: descriptive analytics, predictive analytics and prescriptive analytics (Davenport \& Kim, 2013). These three dimensions answer the following questions: "what happened?", "what is probable to happen?", "what should we do about it? 'In terms of impact, (LaValle et al., 2011) identified that top performing organizations are twice more likely to make decisions which are grounded on strong analyses, for both strategic decisions and operational decisions. Although we have seen that big data can be perceived as a resource, some authors went further, arguing that in order to obtain a positive outcome, an organization has to develop a big data analytics capability. A capability is a unique mix of resources, which makes it difficult, if not impossible, for competitors to imitate it. Within the management literature this would be called gaining a competitive advantage. One such theory is the Resource-Based Theory proposed by Barney (1991), based on which many of the studies included in our meta-analysis are designed (Akter et al., 2016; Shan et al., 2019; Yu et al., 2018).On the same framework, Gupta and George (2016) empirically demonstrated that big data analytics capability enables firms to improve their performance.

\subsection{Performance measurement}

For the performance part, which is the second part of our equation, we identified that the majority of articles are aligned when it comes to measurements methods, but besides this, there is a good amount of diversity in methods. For the most part, the effectiveness of big data analytics is assessed in terms of financial performance, meaning the researches are looking at classical indicators such as: return on assets, return on equity, labor productivity, inventory turnover, profit margin, leverage etc. (Corte-Real et al.,2017; Huang et al., 2018; Raguseo \& Vitari, 2018; Tang et al., 2018; Torres et al., 2018;). Operational performance is very common among studies, meaning the efficiency of processes within firms (Aydiner et al., 2019; Gupta et al., 2018; Fink et al., 2017). Another measurement is that of market performance in comparison with that of competitors (Gupta \& George, 2016; Ren et al., 2016; Wamba et al., 2017;). Besides this, we identified other measurements such as customer retention (Akter et al., 2016), customer satisfaction (Raman et al., 2018), or customer relationship management performance (Nam et al., 2019). One type of performance is that in regards to decision effectiveness. This category is expected when we think that most of the analytics activity has as primal scope to provide decision makers with relevant insights (Cao et al., 2015; Ghasemaghaei et al., 2017; Wang and Byrd, 2017). So far, we tried to make a short description of how big data analytics and performance are treated within the literature we included in our study. But as a more comprehensive and structured analysis we prepared Table 1., where we extracted, out of all the 37 articles included in our meta-analysis, the main takeaways in terms of: samples and unit of analysis, methodologies, operationalizing big data analytics practices, operationalization of performance and the main findings. 
Table 1. Summary of the articles included in the meta-analysis

\begin{tabular}{|c|c|c|c|c|c|}
\hline Paper & $\begin{array}{c}\text { Sample and } \\
\text { unit of analysis }\end{array}$ & Method & $\begin{array}{c}\text { Operationalizing BDA } \\
\text { practices }\end{array}$ & $\begin{array}{c}\text { Operationalization } \\
\text { of performance }\end{array}$ & Findings \\
\hline $\begin{array}{l}\text { (Akter et al., } \\
\text { 2016) }\end{array}$ & 152 responses & $\begin{array}{l}\text { Partial least squares } \\
\text { (PLS) path modeling, } \\
\text { and confirmatory factor } \\
\text { analysis (CFA) }\end{array}$ & $\begin{array}{l}\text { BDA management } \\
\text { capability; BDA technology } \\
\text { capability; BDA talent } \\
\text { capability. }\end{array}$ & $\begin{array}{l}\text { Customer retention, sales } \\
\text { growth, profitability, } \\
\text { return on investment }\end{array}$ & $\begin{array}{l}\text { BDA capability on firm performance } \\
\beta=0.709\end{array}$ \\
\hline $\begin{array}{l}\text { (Anwar et al., } \\
\text { 2018) }\end{array}$ & 312 firms & $\begin{array}{l}\text { Structural Equation } \\
\text { Modeling (SEM) and } \\
\text { CFA }\end{array}$ & $\begin{array}{l}\text { BDA technological; } \\
\text { capabilities; BDA personnel } \\
\text { capabilities. }\end{array}$ & $\begin{array}{l}\text { (1) Competitive } \\
\text { advantage (CA) } \\
\text { (2) Firm Performance } \\
\text { (FP) }\end{array}$ & $\begin{array}{l}\text { BDTC and BDPC have significant } \\
\text { positive impact on FP and CA }\end{array}$ \\
\hline $\begin{array}{l}\text { (Aydiner et al., } \\
\text { 2019) }\end{array}$ & 204 responses & $\begin{array}{l}\text { CFA and SEM using } \\
\text { AMOS }\end{array}$ & $\begin{array}{l}\text { Adoption of business } \\
\text { analytics }\end{array}$ & $\begin{array}{l}\text { (1) Business process } \\
\text { performance (BPER) } \\
\text { (2) Firm performance } \\
\end{array}$ & $\begin{array}{l}\text { Adoption of business analytics } \\
\text { positively influences both BPER and } \\
\text { FP. }\end{array}$ \\
\hline (Cao et al., 2015) & 740 responses & PLS-SEM & Data driven decision making & $\begin{array}{l}\text { Decision making } \\
\text { effectiveness }\end{array}$ & $\begin{array}{l}\text { Business analytics has a positive effect } \\
\text { on decision-making effectiveness. }\end{array}$ \\
\hline $\begin{array}{l}\text { (Chavez et al., } \\
\text { 2017) }\end{array}$ & $\begin{array}{l}329 \text { useable } \\
\text { questionnaires }\end{array}$ & SEM with AMOS 23 & Data-driven supply chains & $\begin{array}{l}\text { Manufacturing } \\
\text { capabilities: Quality, } \\
\text { Delivery, Flexibility, } \\
\text { Cost }\end{array}$ & $\begin{array}{l}\text { The path coefficients are as it follows: } \\
\text { Flexibility (0.493), Delivery }(0.610) \text {, } \\
\text { Quality }(0.526), \text { Cost }(0.599) \text {. }\end{array}$ \\
\hline $\begin{array}{l}\text { (Chen et al., } \\
\text { 2015) }\end{array}$ & Sample of 161 & PLS-SEM & BDA Use & $\begin{array}{l}\text { Asset Productivity } \\
\text { Business Growth }\end{array}$ & $\begin{array}{l}\text { BDA Use on Asset } \beta=0.291 \text {, while } \\
\text { upon the Business Growth } \beta=0.303 \text {. }\end{array}$ \\
\hline $\begin{array}{l}\text { (Corte-Real et } \\
\text { al., 2017) }\end{array}$ & $\begin{array}{l}175 \text { usable } \\
\text { responses }\end{array}$ & PLS methodology & Agility capability & $\begin{array}{l}\text { Financial performance; } \\
\text { Strategic performance. }\end{array}$ & $\begin{array}{l}\text { BDA can enable organizational agility } \\
\text { which impacts competitive advantage. }\end{array}$ \\
\hline $\begin{array}{l}\text { (Côrte-Real et } \\
\text { al., 2019) }\end{array}$ & 175 surveys & PLS & $\begin{array}{l}\text { Strategic alignment between } \\
\text { IT and business, strategic } \\
\text { role of BDA, BDA use. }\end{array}$ & $\begin{array}{l}\text { Strategic and Financial } \\
\text { Performance }\end{array}$ & $\begin{array}{l}\text { BDA use has a positive significant } \\
\text { impact on BDA sustained value, } \\
\beta=0.316 .\end{array}$ \\
\hline $\begin{array}{l}\text { (Dubey et al., } \\
\text { 2017) }\end{array}$ & $\begin{array}{l}205 \\
\text { questionnaires }\end{array}$ & $\begin{array}{l}\text { Ordinary least squares } \\
(\text { OLS) regression }\end{array}$ & $\begin{array}{l}\text { BDA capability: tangible } \\
\text { resources; human resource; } \\
\text { intangible resources. }\end{array}$ & $\begin{array}{l}\text { "Swift trust" impacts } \\
\text { visibility and } \\
\text { coordination. }\end{array}$ & $\begin{array}{l}\text { It has been identified that swift trust } \\
\text { does not have a significant impact on } \\
\text { the relationships between BDPA and } \\
\text { visibility and coordination. }\end{array}$ \\
\hline $\begin{array}{l}\text { (Ferraris et al., } \\
\text { 2018) }\end{array}$ & $\begin{array}{l}88 \text { usable } \\
\text { surveys }\end{array}$ & $\begin{array}{l}\text { SEM within the } \\
\text { resource-based theory } \\
\text { framework. }\end{array}$ & $\begin{array}{l}\text { BDA management and BDA } \\
\text { technological capabilities. }\end{array}$ & Financial performance & $\begin{array}{l}\text { Developing BDA capabilities improved } \\
\text { firms' financial performance. }\end{array}$ \\
\hline $\begin{array}{l}\text { (Fink et al., } \\
\text { 2017) }\end{array}$ & $\begin{array}{l}159 \\
\text { questionnaires }\end{array}$ & SEM. & $\begin{array}{l}\text { Operational business } \\
\text { intelligence and strategic BI } \\
\text { capabilities. }\end{array}$ & $\begin{array}{l}\text { Operational value; } \\
\text { Strategic value. }\end{array}$ & $\begin{array}{l}\text { Operational BI on operational value } \\
\beta=0.518 \text { and strategic BI on strategic } \\
\text { value } \beta=0.186 .\end{array}$ \\
\hline $\begin{array}{l}\text { Ghasemaghaei } \\
(2019)\end{array}$ & $\begin{array}{l}179 \\
\text { questionnaires }\end{array}$ & SEM in SmartPLS 3.0 & $\begin{array}{l}\text { Structural Readiness; } \\
\text { Psychological Readiness. }\end{array}$ & $\begin{array}{l}\text { Value creation: capability } \\
\text { to overcome threats and } \\
\text { exploit opportunities. }\end{array}$ & $\begin{array}{l}\text { BDA use positively influences firm } \\
\text { value creation }(\beta=0.553) \text {, structural } \\
\text { readiness influences BDA use }\end{array}$ \\
\hline
\end{tabular}




\begin{tabular}{|c|c|c|c|c|c|}
\hline Paper & \begin{tabular}{|c|} 
Sample and \\
unit of analysis
\end{tabular} & Method & $\begin{array}{c}\text { Operationalizing BDA } \\
\text { practices }\end{array}$ & $\begin{array}{c}\text { Operationalization } \\
\text { of performance }\end{array}$ & Findings \\
\hline & & & & & $\begin{array}{l}(\beta=0.539, \text { and firm's psychological } \\
\text { readiness BDA use }(\beta=0.161)\end{array}$ \\
\hline $\begin{array}{l}\text { (Ghasemaghaei } \\
\text { et al., 2017) }\end{array}$ & 151 responses & $\begin{array}{l}\text { SmartPLS version } 2.0 \\
\text { and bootstrapping with } \\
500 \text { re-samples }\end{array}$ & BDA capability & $\begin{array}{l}\text { (1) decision quality } \\
\text { (2) decision efficiency }\end{array}$ & $\begin{array}{l}\text { A significant, positive relationship } \\
\text { between data analytics capability and } \\
\text { firm decision-making performance. }\end{array}$ \\
\hline $\begin{array}{l}\text { (Ghasemaghaei } \\
\text { et al., 2017) }\end{array}$ & 215 surveys & $\begin{array}{l}\text { SmartPLS version } 3.0 \\
\text { with bootstrapping } \\
\text { employing } 500 \\
\text { resamples. }\end{array}$ & $\begin{array}{l}\text { BDA use data tools fit, } \\
\text { people tools fit, task tools fit. }\end{array}$ & Firm Agility & $\begin{array}{l}\text { The proposed model has been found to } \\
\text { be non-significant, with a path } \\
\text { coefficient model of }-0.108 \text {. }\end{array}$ \\
\hline $\begin{array}{l}\text { (Gunasekaran et } \\
\text { al., 2016) }\end{array}$ & 205 responses & $\begin{array}{l}\text { Multiple regression } \\
\text { analysis }\end{array}$ & $\begin{array}{l}\text { Connectivity, information } \\
\text { sharing, BDPA Acceptance, } \\
\text { BDPA Assimilation. }\end{array}$ & $\begin{array}{l}\text { Organizational } \\
\text { performance }(\mathrm{OP}) \\
\text { Supply chain } \\
\text { performance }(\mathrm{SCP})\end{array}$ & $\begin{array}{l}\text { Connectivity and information sharing } \\
\text { are positively related to BDPA } \\
\text { acceptance, which is positively related } \\
\text { to BDPA assimilation and positively } \\
\text { related to OP and SCP }\end{array}$ \\
\hline $\begin{array}{l}\text { Gupta and } \\
\text { George (2016) }\end{array}$ & 340 responses & $\begin{array}{c}\text { Exploratory factor } \\
\text { analysis using principal } \\
\text { component analysis and } \\
\text { varimax rotation. } \\
\end{array}$ & $\begin{array}{l}\text { BDA capability:tangible } \\
\text { resources; human resources; } \\
\text { intangible resources. }\end{array}$ & $\begin{array}{l}\text { Operating performance } \\
\text { (OP) Market } \\
\text { performance (MP). }\end{array}$ & For MP $\beta=0.86$, for $\mathrm{OP}, \beta=0.67$. \\
\hline $\begin{array}{l}\text { (Gupta et al., } \\
\text { 2018) }\end{array}$ & $\begin{array}{l}231 \\
\text { questionnaires }\end{array}$ & $\begin{array}{l}\text { PLS-SEM. by Warp } \\
\text { PLS version } 6.0 \text {. }\end{array}$ & $\begin{array}{l}\text { BDA capability: data, } \\
\text { managerial skills, technical } \\
\text { skills. }\end{array}$ & $\begin{array}{l}\text { Market performance } \\
\text { (MP); Operational } \\
\text { performance (OP) }\end{array}$ & $\begin{array}{l}\text { BDPA has a positive effect on MP } \\
(\beta=0.51) \text {. } \\
\text { BDPA has a positive effect on OP } \\
(\beta=0.48) .\end{array}$ \\
\hline \begin{tabular}{|l} 
Hosoya and \\
Kamioka (2018)
\end{tabular} & 107 surveys & $\begin{array}{l}\text { The hypotheses have } \\
\text { been tested using SEM. }\end{array}$ & $\begin{array}{l}\text { Ad Hoc use of BDA, } \\
\text { interpretation, learning. }\end{array}$ & $\begin{array}{l}\text { Sensemaking, agility, } \\
\text { firm performance }\end{array}$ & Agility on firm performance, $\beta=0.72$. \\
\hline $\begin{array}{l}\text { (Huang et al., } \\
\text { 2018) }\end{array}$ & 386 surveys & OLS regression models & BDA implementations. & $\begin{array}{l}\text { Financial performance, } \\
\text { productivity, market } \\
\text { valuation. }\end{array}$ & $\begin{array}{l}\text { BDA implementation positively affects } \\
\text { financial performance and market } \\
\text { valuation, but not productivity. }\end{array}$ \\
\hline $\begin{array}{l}\text { (Jeble et al., } \\
2018)\end{array}$ & 205 responses & PLS-SEM & BDA Capability & $\begin{array}{l}\text { Environmental, } \\
\text { Social, and } \\
\text { Economic Performance } \\
\end{array}$ & $\begin{array}{l}\text { Environmental Performance }(\beta=0.74), \\
\text { Social Performance }(\beta=0.21) \text {, Economic } \\
\text { Performance }(\beta=0.80) \text {. }\end{array}$ \\
\hline Mandal (2018) & $\begin{array}{l}249 \\
\text { questionnaires }\end{array}$ & $\begin{array}{l}\text { PLS in SmartPLS } \\
\text { 2.0.M3 }\end{array}$ & $\begin{array}{l}\text { BDA management } \\
\text { capabilities. }\end{array}$ & $\begin{array}{l}\text { Supply chain } \\
\text { preparedness, alertness } \\
\text { and agility }\end{array}$ & $\begin{array}{l}\text { BDA planning, BDA coordination and } \\
\text { control BDA have a positive effect. } \\
\text { BDA investment decision making does } \\
\text { not have a significant impact. }\end{array}$ \\
\hline $\begin{array}{l}\text { (Müller et al., } \\
\text { 2018) }\end{array}$ & $\begin{array}{l}\begin{array}{l}\text { Panel } 814 \text { firms } \\
\text { during period } \\
2008-2014\end{array} \\
\end{array}$ & $\begin{array}{l}\text { OLS; fixed-effects } \\
\text { estimator and; fixed- } \\
\text { effects two-stage least } \\
\text { squares }\end{array}$ & $\begin{array}{l}\text { BDA assets: labor, capital, } \\
\text { material }\end{array}$ & Firm productivity (Sales) & $\begin{array}{l}\text { BDA assets are associated with an } \\
\text { average of 3-7\% improvement in } \\
\text { firm's productivity }\end{array}$ \\
\hline
\end{tabular}




\begin{tabular}{|c|c|c|c|c|c|}
\hline Paper & $\begin{array}{c}\text { Sample and } \\
\text { unit of analysis }\end{array}$ & Method & $\begin{array}{c}\text { Operationalizing BDA } \\
\text { practices }\end{array}$ & $\begin{array}{c}\text { Operationalization } \\
\text { of performance }\end{array}$ & Findings \\
\hline $\begin{array}{l}\text { (Nam et al., } \\
\text { 2019) }\end{array}$ & Sample of 170 & PLS method & $\begin{array}{l}\text { IT competence, data } \\
\text { management capability. }\end{array}$ & $\begin{array}{l}\text { CRM performance, } \\
\text { mediated by customer } \\
\text { response capability }\end{array}$ & $\begin{array}{l}\text { Customer response capability mediates } \\
\text { between business analytics use and } \\
\text { CRM performance. }\end{array}$ \\
\hline $\begin{array}{l}\text { (Niebel et al., } \\
\text { 2019) }\end{array}$ & 2706 firms & $\begin{array}{l}\text { Probit model, Tobit } \\
\text { model, fractional logit } \\
\text { model. }\end{array}$ & $\begin{array}{l}\text { BDA is digitized information } \\
\text { and analytical technologies. }\end{array}$ & $\begin{array}{l}\text { Percentage of sales } \\
\text { resulted from new } \\
\text { products/services. }\end{array}$ & $\begin{array}{l}\text { BDA increases tendency to innovate by } \\
6.7 \% \text {. }\end{array}$ \\
\hline $\begin{array}{l}\text { (Popovič et al., } \\
\text { 2018) }\end{array}$ & $\begin{array}{l}181 \\
\text { questionnaires }\end{array}$ & PLS-SEM & $\begin{array}{l}\text { Routine use and innovative } \\
\text { use of business intelligence } \\
\text { systems }\end{array}$ & $\begin{array}{l}\text { Marketing and sales; } \\
\text { management and internal } \\
\text { operations; procurement. }\end{array}$ & $\begin{array}{l}\text { Marketing and sales } \beta=0.254, \\
\text { management and internal operations } \\
\beta=0.580, \text { procurement } \beta=0.017 \text {. }\end{array}$ \\
\hline $\begin{array}{l}\text { Raguseo and } \\
\text { Vitari (2018) }\end{array}$ & $\begin{array}{l}76 \\
\text { questionnaires }\end{array}$ & Variance-based SEM & $\begin{array}{l}\text { Business value BDA } \\
\text { solutions. }\end{array}$ & $\begin{array}{l}\text { Financial performance } \\
\text { Market performance } \\
\text { Customer satisfaction }\end{array}$ & $\begin{array}{l}\text { BDA solutions explains } 62.4 \% \text { of the } \\
\text { variance of customer satisfaction, } \\
71.9 \% \text { of the variance of market } \\
\text { performance, and } 78.6 \% \text { of the variance } \\
\text { of financial performance. }\end{array}$ \\
\hline $\begin{array}{l}\text { (Raman et al., } \\
\text { 2018) }\end{array}$ & 287 responses & SEM & $\begin{array}{l}\text { Demand management; } \\
\text { vendor rating; analytics; } \\
\text { IOT; data science. }\end{array}$ & $\begin{array}{l}\text { Supply chain } \\
\text { management } \\
\text { performance }\end{array}$ & $\begin{array}{l}\text { BDA practices have a positive effect on } \\
\text { supply chain management } \\
\text { effectiveness. }\end{array}$ \\
\hline (Ren et al., 2016) & \begin{tabular}{|l|}
287 \\
questionnaires
\end{tabular} & PLS-SEM & $\begin{array}{l}\text { BDA system quality, } \\
\text { BDA information quality. }\end{array}$ & $\begin{array}{l}\text { Financial Performance; } \\
\text { Market Performance }\end{array}$ & $\begin{array}{l}\text { BDA business value upon firm } \\
\text { performance, } \beta=0.28 \text {. }\end{array}$ \\
\hline $\begin{array}{l}\text { (Shan et al., } \\
\text { 2019) }\end{array}$ & $\begin{array}{l}219 \\
\text { questionnaires }\end{array}$ & CFA and SEM & $\begin{array}{l}\text { IT technology resources; } \\
\text { IT relationship resources; } \\
\text { Idle resources. }\end{array}$ & Competitive advantage & $\begin{array}{l}\text { The hypotheses for IT technology } \\
\text { capabilities and compatibility are } \\
\text { supported }(\beta=0.179 \text { and } 0.351) \text {, while } \\
\text { the hypothesis for strategy flexibility's } \\
\text { impact on competitive advantage is not } \\
\text { supported }(\beta=0.001) \text {. }\end{array}$ \\
\hline Singh (2019) & $\begin{array}{l}215 \\
\text { questionnaires }\end{array}$ & $\begin{array}{c}\text { PLS-SEM in Smart PLS } \\
3\end{array}$ & $\begin{array}{l}\text { Corporate commitment, } \\
\text { big data acceptance, } \\
\text { big data assimilation. }\end{array}$ & \begin{tabular}{|l|} 
Environmental \\
Performance \\
Organizational \\
Performance \\
\end{tabular} & $\begin{array}{l}\text { The path coefficient for Big Data } \\
\text { Assimilation impact on OP is } 0.253 \text {. }\end{array}$ \\
\hline $\begin{array}{l}\text { (Song et al., } \\
\text { 2018) }\end{array}$ & 309 responses & $\begin{array}{l}\text { CFA and OLS, as well } \\
\text { as correlation matrix } \\
\text { and regression analysis }\end{array}$ & $\begin{array}{l}\text { BDA usage toward Demand- } \\
\text { side; } \\
\text { BDA usage toward Supply- } \\
\text { side. }\end{array}$ & $\begin{array}{l}\text { Performance of the } \\
\text { merchants }\end{array}$ & $\begin{array}{l}\text { Both demand-side and supply-side data } \\
\text { analytics usage have a positive impact } \\
\text { on the performance of merchants, } \\
\beta=0.112 \text { and } 0.072 \text {. }\end{array}$ \\
\hline $\begin{array}{l}\text { (Tang et al., } \\
\text { 2018) }\end{array}$ & 432 firms & OLS regression. & $\begin{array}{l}\text { RFID, wireless sensor } \\
\text { networks, middleware, cloud } \\
\text { computing, and IoT } \\
\text { application software. }\end{array}$ & $\begin{array}{l}\text { Financial performance } \\
\text { Productivity } \\
\text { Market value }\end{array}$ & $\begin{array}{l}\text { A positive impact of IoT on all three } \\
\text { dimensions of performance. }\end{array}$ \\
\hline
\end{tabular}




\begin{tabular}{|c|c|c|c|c|c|}
\hline Paper & $\begin{array}{c}\text { Sample and } \\
\text { unit of analysis }\end{array}$ & Method & $\begin{array}{c}\text { Operationalizing BDA } \\
\text { practices }\end{array}$ & $\begin{array}{c}\text { Operationalization } \\
\text { of performance }\end{array}$ & Findings \\
\hline $\begin{array}{l}\text { (Torres et al., } \\
\text { 2018) }\end{array}$ & 137 surveys & PLS-SEM & $\begin{array}{l}\text { Business Intelligence and } \\
\text { Analytics }\end{array}$ & $\begin{array}{l}\text { Operational performance } \\
\text { Financial performance }\end{array}$ & $\begin{array}{l}\text { A positive relationship between } \\
\text { Business Intelligence and Analytics and } \\
\text { Firm Performance. }\end{array}$ \\
\hline $\begin{array}{l}\text { (Wamba et al., } \\
\text { 2017) }\end{array}$ & Sample of 297 & PLS-SEM & $\begin{array}{l}\text { BDA infrastructure } \\
\text { flexibility; BDA } \\
\text { management capabilities, } \\
\text { BDA personnel expertise } \\
\text { capability. }\end{array}$ & $\begin{array}{l}\text { Firm performance } \\
\text { (FPER) is divided into } \\
\text { financial performance } \\
\text { and market performance. }\end{array}$ & $\begin{array}{l}\text { BDA capability has a significant } \\
\text { positive impact on FPER with a path } \\
\text { coefficient of } 0.56 .\end{array}$ \\
\hline $\begin{array}{l}\text { (Wamba et al., } \\
\text { 2018) }\end{array}$ & $\begin{array}{l}302 \text { business } \\
\text { analysts }\end{array}$ & $\begin{array}{l}\text { PLS-SEM and REBUS- } \\
\text { PLS algorithm }\end{array}$ & $\begin{array}{l}\text { Information Quality: } \\
\text { completeness, currency, } \\
\text { format and accuracy. }\end{array}$ & $\begin{array}{l}\text { Firm Performance is } \\
\text { constructed on Business } \\
\text { Value and User } \\
\text { Satisfaction. }\end{array}$ & $\begin{array}{l}\text { Information quality has significant, } \\
\text { positive impact on firm performance. }\end{array}$ \\
\hline $\begin{array}{l}\text { Wang and Byrd } \\
(2017)\end{array}$ & 152 responses & CFA and SEM & Absorptive capacity & $\begin{array}{l}\text { Decision-making } \\
\text { effectiveness }\end{array}$ & $\begin{array}{l}\text { Business analytics capabilities improve } \\
\text { absorptive capacity, which in turn } \\
\text { positively impact the decision-making } \\
\text { effectiveness }(\beta=0.441) \text {. }\end{array}$ \\
\hline (Yu et al., 2018) & $\begin{array}{l}329 \\
\text { questionnaires }\end{array}$ & SEM with AMOS 23 & Data-driven supply chains & $\begin{array}{l}\text { Information exchange; } \\
\text { Coordination; Activity } \\
\text { integration, } \\
\text { Responsiveness. }\end{array}$ & $\begin{array}{l}\text { Information Exchange }(\beta=0.765) \\
\text { Coordination }(\beta=0.880) \text {, Activity } \\
\text { Integration }(\beta=0.878), \text { Responsiveness } \\
(\beta=0.900) \text {. }\end{array}$ \\
\hline
\end{tabular}

Source:Own editing, based on the literature reviewed 


\section{RESEARCH METHODOLOGY}

The main scope of our study is to assess the extent to which big data analytics produces positive effects within a firm. A meta-analysis is suitable for our need because this is a quantitative method to merge data from independent studies, which are treating the same topic. As a basis, we used the results of all the articles we managed to find which analyzed a form of big data analytics and the outcome it produces in terms of performance. The rationale for doing a meta-analysis is indicated by several advantages of this methodology such as: increased power of the study, as it can identify significant differences between study groups; combined sample of all the studies, which improves precision; tests the hypotheses across study groups, with different characteristics offering the possibility to identify clusters, and finally, it makes possible to compare conflicting studies, so that the discrepancies can be settled (Moore, 2012). We followed the procedures recommended by specialist from the field of social sciences. A meta-analysis should follow a couple of steps: a comprehensive literature search, a clear criterion for the inclusion of an article in the study, calculation of effect sizes, and finally running the meta-analysis itself (Field \& Gillett, 2010).

In terms of literature search, we used mainly the ISI Web of Knowledge database, due to the myriad of articles available and on the quality requirements for inclusion in our database. As searching criteria, we used tags such as "big data" or "data science" or "business analytics" combined with "firm performance" or "competitive advantage" or "value". The first selection operation was performed based on title and abstract. After applying these two filters we remained with a database of 375 articles. The next step was the selection based on study type, where we included only the empirical studies, so we eliminated literature review studies and case studies. This was the most rigorous step, which left us with 52 articles. Further on, we did an additional clean up based on the alignment with our topic of the analysis of the articles and the availability of the measurements methodologies and results. Finally, we included 37 articles in our meta-analysis.

The next step in our meta-analysis was the calculation or the extraction of the effect sizes. An effect size is a standardized measure which reflects the magnitude of the observed element in each study (Field \& Gillett, 2010). In our approach, we used the standardized regression coefficient $(\beta)$, which is similar with Cohen's $d$, which is one of the most widely used effect sizes and represents the standardized difference between two means (Baguley, 2019; Grace-Martin, 2011;). As an observation, we would add that where possible, we included more than one path coefficient from the articles. This is applicable when a study is assessing the impact of big data analytics upon multiple dimensions of performance (e.g. operational performance and market performance). Because of this decision, although our meta-analysis is based on 37 articles, the number of effect sizes included is 84 . One of the advantages of a meta-analysis study is the increased power of the study given by the cumulated samples of the individual studies. In this regard, we can say that from our pool of articles, we identified only two cases where two articles shared the same sample. Excluding these two duplications we are left with an aggregated sample of 11389 individual observations. Secondary, in order to assign the weight of each study we calculated the standard error (SE) for each study (Borenstein et al., 2011). 
In our approach we used the sample size of each study based on which we calculated the square root of the variance:

$$
S E=\sqrt{\frac{1}{\text { sample size-3 }}}(\text { Cheung, 2015) (1) }
$$

After obtaining the effect size and the standard error, the next step is to perform the meta-analysis itself. There were two methods that we could have used here: the fixed effect model or the random effects model. The former model assumes that the studies included originate from a homogeneous population, so the larger studies will be assigned a bigger weight (Field \&Gillett, 2010). On the other hand, the latter model assumes that the similarity between studies is just enough so that it makes sense to include them in the same analysis, but besides this, the studies have their own specificities, so their effects are not identical (Borenstein et al., 2011). In our analysis we calculated both models. As a computing environment we used RStudio with $R$ version 3.6.1. R package meta was used with the metagen function. In terms of estimation of between-study variance we used DerSimonian-Laird estimator which is the default method in metagen function and in the same time the only one available in RevMan 5, a specially designed software for meta-analysis. The DerSimonian and Laird estimator is calculated as it follows (Schwarzer et al. 2015):

$$
\hat{\tau}^{2}=\frac{Q-(k-1)}{\sum_{k-1}^{k} \frac{\sum_{k-1}^{k} w_{k}^{2}}{\sum_{k=1}^{k} w_{k}}}
$$

where $\mathrm{Q}$, the heterogeneity statistic is calculated as: $Q=\sum_{k=1}^{k} w_{k}\left(\widehat{\theta_{k}}-\widehat{\theta_{F}}\right)^{2}$ and $w_{k}=$ $\widehat{\operatorname{Var}}\left(\widehat{\theta_{k}}\right)^{-1}$. The estimator $\hat{\tau}^{2}$ is equal to 0 if $Q<K-1$.

\section{RESULTS AND DISCUSSIONS}

\subsection{Findings of the study}

The results of our meta-analysis are visually displayed under Appendix 1. Forest plot for the meta-analysis and Appendix 2. Summarization of results in $R$ Studio. The values of the columns from Appendix 1 indicate the following: Study - author/s and year of publication; $T E$ - effect size of the study, seTE - standard error of the effect size; Log Risk Ratio - is the visual representation of the effect size and its confidence interval on an scale from -1 to $1 ; \log R R$ and $95 \% C I-$ the effect size and its corresponding coefficient interval, Weight (fixed) - the weigh of the study based on fixed effect model, Weight (random) - the weight of the study based on the random effects model.

Based on the fixed effect model, the aggregated value of the effects sizes is 0.4558 with a $95 \%$ confidence interval of $[0.4435 ; 0.4680], p<0.0001$. In the case of random effects model the log risk ratio is 0.4864 with a confidence interval of $[0.4233 ; 0.5496], \mathrm{p}<0.0001$. The reason why the confidence interval is larger in the case of random effects model is because, besides the withinstudy sampling error, there is there is a secondary source of between-studies variance (Borenstein et al., 2011).An important decision is the choice between fixed effect model and random effects model. Because of the algorithm of the metagen function from $\mathrm{R}$, both are calculated by default. 
However, there are statistical imperatives why only one is suitable for our study design. Borenstein et al. (2011) argue that when studies included in meta-analysis are conducted independently it is very unlikely to have a common effect size. Additionally, the use of random effects model gives the possibility to generalize the results from the studied populations to others. This drives us to choose the random effects model. The results confirm that big data analytics have a significant positive impact upon organizational performance with an aggregated path coefficient of 0.4864 ( $p<0.0001)$.Within our literature search, we have identified multiple big data analytics implementations and levels of applicability within firms. Big data analytics is being applied across all levels: fromad-hoc use, to the transformation of a function such as data-driven supply chains, culminating with the developing of a big data analytics capability. Firms invest vast resources and human skills, technology and technical expertise, IT infrastructure and process transformation, managers act as agents of change in order to nurture data focused cultures. These are the requirements and characteristics which an organization has to acquire in order to initiate a big data transformation. It is a meandrous, demanding and challenging path. But this is just one part of the equation. On the other side, we found that the benefits gained make it worth. What we generally identified as performance comes in many shapes. As a first tangible effect is decision effectiveness. This in turn impacts process efficiency in the form of streamlined operations; customer satisfaction because of an improved customer relationship management; sales growth and increased market share; financial performance, improving labor productivity, return on assets, and inventory turnover. In terms of strategic value, we identified benefits such as: firm agility, the firm being able to anticipate and to adapt to changes and eventually capacity to obtain competitive advantages either as cost leadership or as capability to differentiate.

\subsection{Implications for practice}

The potential and the challenge raised by the big data analytics trend are both promising and threatening. On one hand, it offers unprecedented possibilities: to decide based on facts and knowledge as close to reality as possible, to correctly allocate resources, to understand customers and transform organizations. But on the other hand, it involves uncertainty. As seen in the last years, big data analytics combined with automation promise to bring a new industrial revolution. In order to keep the pace, managers have to adopt a decision-making style centered on data and facts, in contrast with the classical style based on experience and intuition. They should act as agents of change. Organizations have to increase the intensity of organizational learning. First of all, it is needed for the organization to acquire the necessary knowledge in order to develop a big data analytics capability, and second to make it possible to create new knowledge with data and to act based on it. In terms of expertise and professions, both employees and managers have to be trained and to develop new skill sets. There is a scarcity of experts, so called data scientists, so organizations face a double challenge. On one hand, how to attract these specialists into the organization, and on the other hand, how to train their employees in order to develop the essential skills for this transformation. Managers alike, have to "pay their dues" as well, because in order to initiate and drive this change, they need to develop a basic analytics acumen, but most importantly to lead by example. This involves changing their management styles to the core, especially in the way they are making decisions. All in all, these changes and challenges bring the organization and the way it functions closer to the principles by which science is governed. That implies using data, evidence and facts and has as final goal the pursuit of truth. 


\section{CONCLUSION}

\subsection{Contribution of the study}

In this study we aimed to quantitatively assess, with the help of a meta-analysis the impact of big data analytics upon organizational performance. We found strong evidence that big data analytics has a positive impact upon organizational performance. The results of the 37 articles, which we included in our study, converge to the conclusion that organizations do benefit, in many forms from a big data initiative. However, in order to adopt to this revolution, resources need to be put in place and employees and managers alike need to develop new sets of skills and behaviors. Our findings help to strengthen and to support the idea that big data analytics transformation is beneficial for an organization.

\subsection{Limitations and future research}

The limitation of the current study set the path for future research opportunities. When doing a meta-analysis, one of the risks involved is missing out relevant studies. One of the reasons for this is publication bias, which comes from the fact that articles with significant favorable results have a higher chance to be published while the other ones get rejected more often (Field and Gillett, 2010).In order to avoid this issue, one measure would be, that the researchers contact the authors of the discovered studies and ask whether or not there were additional trials, where the results found rejected the premise of the study. Impossibility to validate the hypothesis discourages the authors to send for publication the results or could decrease the chances of the study do be accepted.

Second, the design of our study is centered on computing an aggregated score for all the articles included in the meta-analysis. We believe it to be relevant that sub-group analyses are performed, based on criteria such as: sector or size of the firm, big data analytics practice, dimension of performance. This clusterization can help, if found, to isolate the positive effect which we identified, to a specific characteristic either of the firm, the practice, or a dimension of performance

\section{REFERENCES}

Adesina, A. (2018). Data is the new oil. Retreived June 5, 2019 from https://medium.com /@ adeolaadesina/data-is-the-new-oil-2947ed8804f6

Akter, S., Wamba, S. F., Gunasekaran, A., Dubey, R., \& Childe, S. J. (2016). How to improve firm performance using big data analytics capability and business strategy alignment? International Journal of Production Economics, 182, 113-131.

Anwar, M., Khan, S. Z., \& Shah, S. Z. A. (2018). Big Data Capabilities and Firm's Performance: A Mediating Role of Competitive Advantage. Journal of Information \& Knowledge Management, 17(04), 1-28.

Aydiner, A. S., Tatoglu, E., Bayraktar, E., Zaim, S., \&Delen, D. (2019). Business analytics and firm performance: The mediating role of business process performance. Journal of Business Research, 96, 228-237.

Baguley, T. (2009). Standardized or simple effect size: What should be reported? British journal of psychology, 100(3), 603-617.

Barney, J. (1991). Firm resources and sustained competitive advantage. Journal of management, 17(1), 99-120. 
Borenstein, M., Hedges, L. V., Higgins, J. P., \& Rothstein, H. R. (2011). Introduction to metaanalysis. John Wiley \& Sons.

Cao, G., Duan, Y., \& Li, G. (2015). Linking business analytics to decision making effectiveness: A path model analysis. IEEE Transactions on Engineering Management, 62(3), 384-395.

Chen, D. Q., Preston, D. S., \&Swink, M. (2015). How the use of big data analytics affects value creation in supply chain management. Journal of Management Information Systems, 32(4), 4-39.

Cheung, M. W. L. (2015). Meta-analysis: A structural equation modeling approach. John Wiley \& Sons.

Côrte-Real, N., Oliveira, T., \&Ruivo, P. (2017). Assessing business value of Big Data Analytics in European firms. Journal of Business Research, 70, 379-390.

Côrte-Real, N., Ruivo, P., Oliveira, T., \&Popovič, A. (2019). Unlocking the drivers of big data analytics value in firms. Journal of Business Research, 97, 160-173.

Davenport, T. H., \& Harris, J. G. (2007). Competing on analytics: The new science of winning. Harvard Business Press.

Davenport, T. H., \& Kim, J. (2013). Keeping up with the quants: Your guide to understanding and using analytics. Harvard Business Review Press.

Dubey, R., Luo, Z., Gunasekaran, A., Akter, S., Hazen, B. T., \& Douglas, M. A. (2018). Big data and predictive analytics in humanitarian supply chains: Enabling visibility and coordination in the presence of swift trust. The International Journal of Logistics Management, 29(2), 485-512.

Ferraris, A., Mazzoleni, A., Devalle, A., \& Couturier, J. (2018). Big data analytics capabilities and knowledge management: impact on firm performance. Management Decision.

Field, A. P., \& Gillett, R. (2010). How to do a meta-analysis. British Journal of Mathematical and Statistical Psychology, 63(3), 665-694.

Fink, L., Yogev, N., \& Even, A. (2017). Business intelligence and organizational learning: An empirical investigation of value creation processes. Information \& Management, 54(1), 38-56.

FossoWamba, S., Akter, S., Trinchera, L., \& De Bourmont, M. (2018). Turning information quality into firm performance in the big data economy. Management Decision.

Gartner. (n.d.). Big Data, Retrieved June 5, 2019, from https://www.gartner.com/it-glossary/bigdata/

Ghasemaghaei, M., Ebrahimi, S., \& Hassanein, K. (2018). Data analytics competency for improving firm decision making performance. The Journal of Strategic Information Systems,27(1), 101-113.

Ghasemaghaei, M., Hassanein, K., \& Turel, O. (2017). Increasing firm agility through the use of data analytics: The role of fit. Decision Support Systems, 101, 95-105.

Giacaglia, G. (2019). Data is the New Oil. RetrievedJune 5, 2019 from https://hackernoon.com/data-is-the-new-oil-1227197762b2

Glass, G. V. (1978). In defense of generalization. Behavioral and Brain Sciences, 1(3), 394-395.

Grace-Martin, K. (2011). A comparison of effect size statistics. The Analysis Factor. Retrieved on June 20 from https://www.theanalysisfactor.com/effect-size/

Gunasekaran, A., Papadopoulos, T., Dubey, R., Wamba, S. F., Childe, S. J., Hazen, B., \& Akter, S. (2017). Big data and predictive analytics for supply chain and organizational performance. Journal of Business Research, 70, 308-317.

Gupta, M., \& George, J. F. (2016). Toward the development of a big data analytics capability. Information \& Management, 53(8), 1049-1064. 
Gupta, S., Qian, X., Bhushan, B., \& Luo, Z. (2018). Role of cloud ERP and big data on firm performance: a dynamic capability view theory perspective. Management Decision.

Hosoya, R., \&Kamioka, T. (2018, August). Understanding how the ad hoc use of big data analytics impacts agility: a sense making-based model. In 2018 International Conference on Advances in Big Data, Computing and Data Communication Systems (icABCD) (pp. 1-8). IEEE.

Huang, C. K., Wang, T., \& Huang, T. Y. (2018). Initial Evidence on the Impact of Big Data Implementation on Firm Performance. Information Systems Frontiers, 1-13.

Jeble, S., Dubey, R., Childe, S. J., Papadopoulos, T., Roubaud, D., \& Prakash, A. (2018). Impact of big data and predictive analytics capability on supply chain sustainability. The International Journal of Logistics Management, 29(2), 513-538.

Ji-fan Ren, S., FossoWamba, S., Akter, S., Dubey, R., \& Childe, S. J. (2017). Modelling quality dynamics, business value and firm performance in a big data analytics environment. International Journal of Production Research, 55(17), 5011-5026.

LaValle, S., Lesser, E., Shockley, R., Hopkins, M. S., \& Kruschwitz, N. (2011). Big data, analytics and the path from insights to value. MIT sloan management review, 52(2), 21-32.

Mandal, S. (2019). The influence of big data analytics management capabilities on supply chain preparedness, alertness and agility: An empirical investigation. Information Technology \& People, 32(2), 297-318.

Meyerson, D., Weick, K. E., \& Kramer, R. M. (1996). Swift trust and temporary groups. Trust in organizations: Frontiers of theory and research, 166, 195.

Moore, Z. (2012). Meta-analysis in context. Journal of clinical nursing, 21(19pt20), 2798-2807.

Müller, O., Fay, M., \& vomBrocke, J. (2018). The effect of big data and analytics on firm performance: An econometric analysis considering industry characteristics. Journal of Management Information Systems, 35(2), 488-509.

Nam, D., Lee, J., \& Lee, H. (2019). Business analytics use in CRM: A nomological net from IT competence to CRM performance. International Journal of Information Management, 45, 233-245.

Niebel, T., Rasel, F., \&Viete, S. (2019). BIG data-BIG gains? Understanding the link between big data analytics and innovation. Economics of Innovation and New Technology, 28(3), 296-316.

Popovič, A., Puklavec, B., \& Oliveira, T. (2019). Justifying business intelligence systems adoption in SMEs: Impact of systems use on firm performance. Industrial Management \& Data Systems, 119(1), 210-228.

Raguseo, E., \&Vitari, C. (2018). Investments in big data analytics and firm performance: an empirical investigation of direct and mediating effects. International Journal of Production Research, 56(15), 5206-5221.

Raman, S., Patwa, N., Niranjan, I., Ranjan, U., Moorthy, K., \& Mehta, A. (2018). Impact of big data on supply chain management. International Journal of Logistics Research and Applications, 21(6), 579-596.

Rosenthal, R. (1991). Meta-Analytic Procedures for Social Research. Sage Publications, Inc.

Schwarzer, G., Carpenter, J. R., \&Rücker, G. (2015). Meta-analysis with $R$ (pp. 28-36). New York, NY: Springer.

Shan, S., Luo, Y., Zhou, Y., \& Wei, Y. (2019). Big data analysis adaptation and enterprises' competitive advantages: the perspective of dynamic capability and resource-based theories. Technology Analysis \& Strategic Management, 31(4), 406-420. 
Singh, S. K., \& El-Kassar, A. N. (2019). Role of big data analytics in developing sustainable capabilities. Journal of cleaner production, 213, 1264-1273.

Song, P., Zheng, C., Zhang, C., \& Yu, X. (2018). Data analytics and firm performance: An empirical study in an online B2C platform. Information \& Management, 55(5), 633-642.

Tang, C. P., Huang, T. C. K., \& Wang, S. T. (2018). The impact of Internet of things implementation on firm performance. Telematics and Informatics, 35(7), 2038-2053.

The Economist. (2017). The world's most valuable resource is no longer oil, but data. RetrievedJune 5 2019, fromhttps://www.economist.com/leaders/2017/05/06/the-worldsmost-valuable-resource-is-no-longer-oil-but-data

Torres, R., Sidorova, A., \& Jones, M. C. (2018). Enabling firm performance through business intelligence and analytics: A dynamic capabilities perspective. Information \& Management, 55(7), 822-839.

Wamba, S. F., Gunasekaran, A., Akter, S., Ren, S. J. F., Dubey, R., \& Childe, S. J. (2017). Big data analytics and firm performance: Effects of dynamic capabilities. Journal of Business Research, 70, 356-365.

Wang, Y., \& Byrd, T. A. (2017). Business analytics-enabled decision-making effectiveness through knowledge absorptive capacity in health care. Journal of Knowledge Management, 21(3), 517-539.

Yu, W., Chavez, R., Jacobs, M. A., \& Feng, M. (2018). Data-driven supply chain capabilities and performance: A resource-based view. Transportation Research Part E: logistics and transportation review, 114, 371-385. 
APPENDIX 1. FOREST PLOT FOR THE META-ANALYSIS

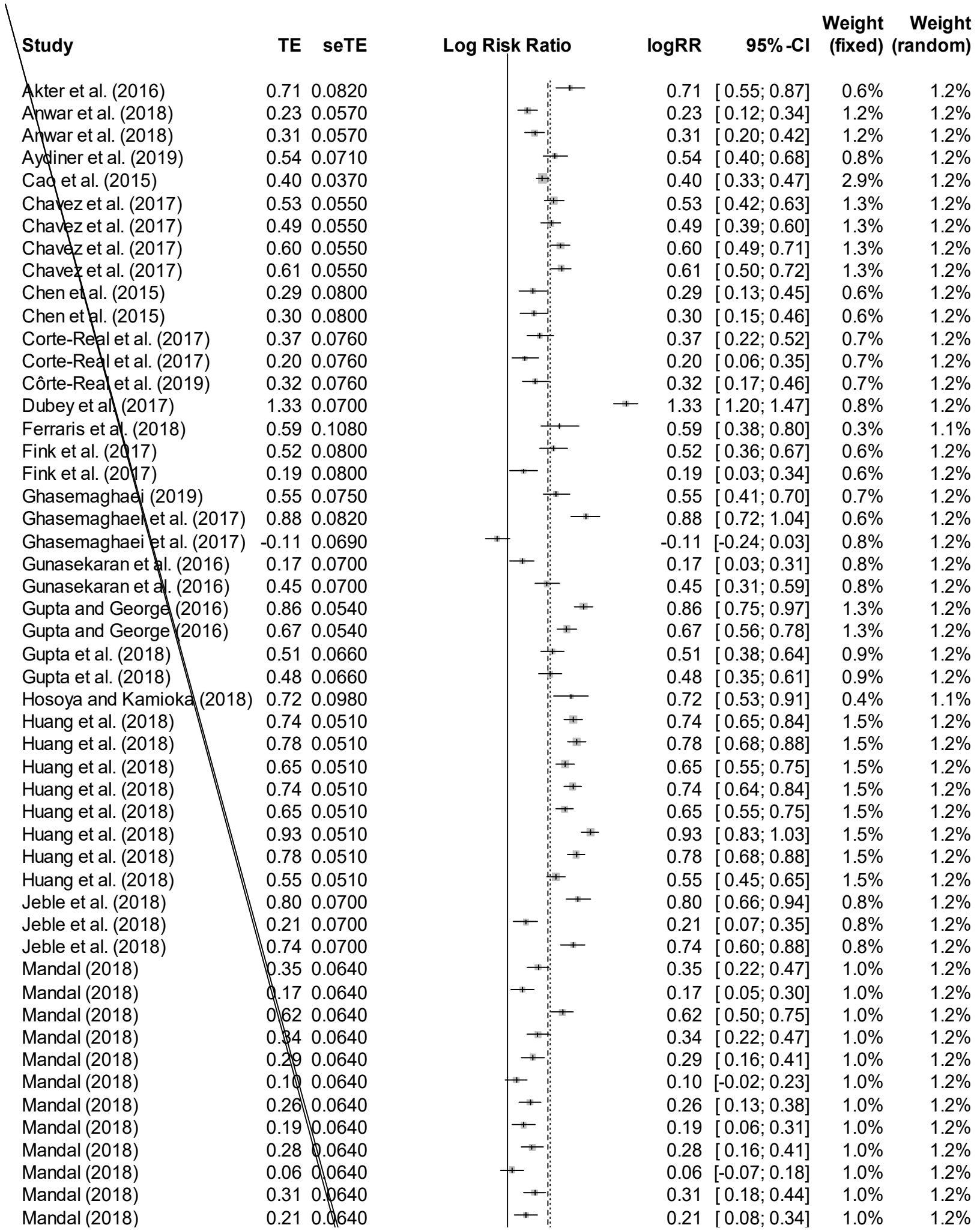




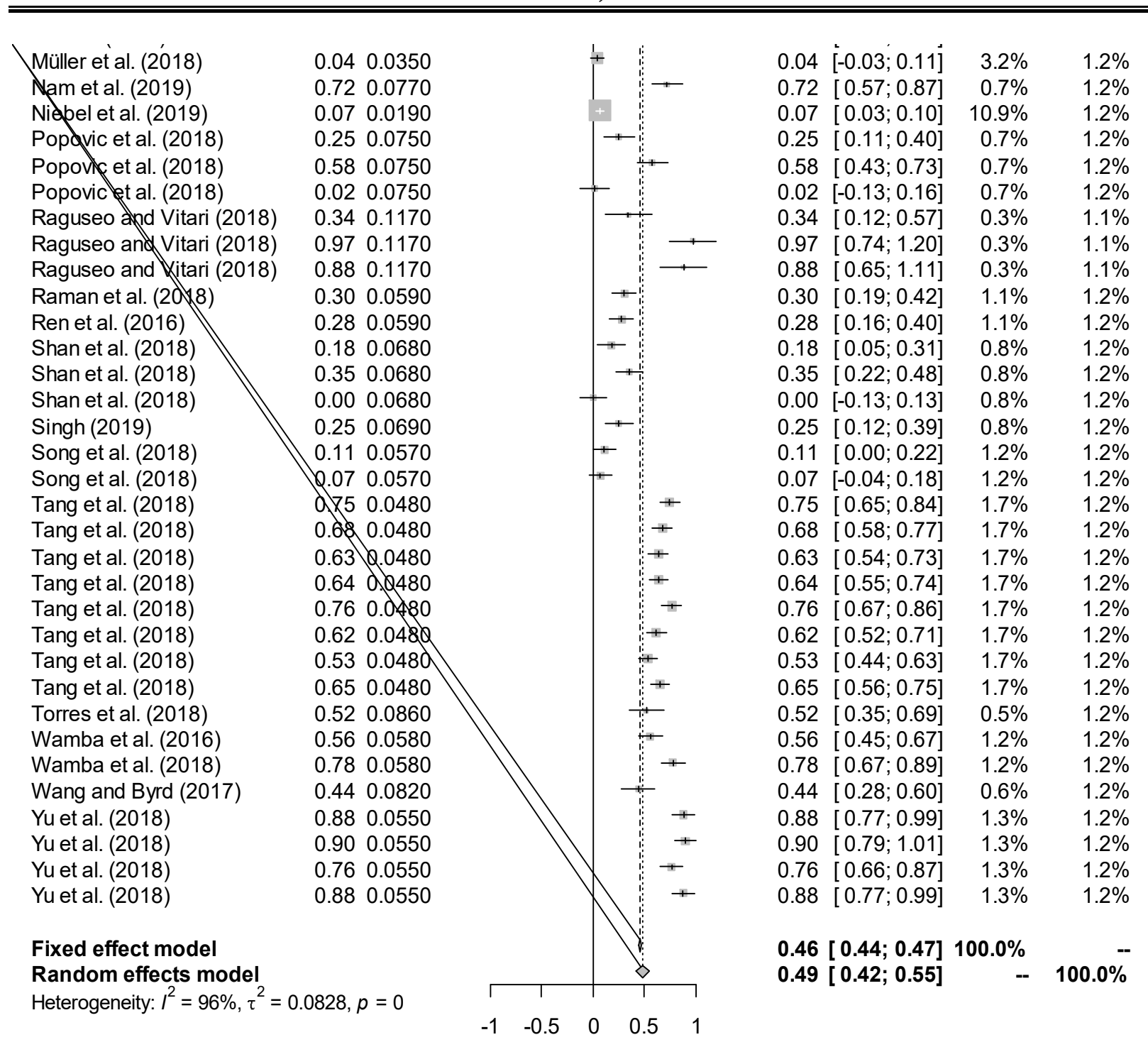

\section{APPENDIX 2. SUMMARIZATION OF RESULTS IN R STUDIO}

Number of studies combined: $\mathrm{k}=84$

$\operatorname{logRR} \quad 95 \%$-CI $\mathrm{z}$ p-value

Fixed effect model $0.4558[0.4435 ; 0.4680] 72.77 \quad 0$

Random effects model 0.4864 [0.4233; 0.5496] $15.09<0.0001$

Quantifying heterogeneity:

$\operatorname{tau}^{\wedge} 2=0.0828 ; \mathrm{H}=5.08$ [4.78; 5.40]; $\mathrm{I}^{\wedge} 2=96.1 \%$ [95.6\%; 96.6\%]

Test of heterogeneity:

Q d.f. p-value

$2144.94 \quad 83 \quad 0$

Details on meta-analytical method:

- Inverse variance method

- DerSimonian-Laird estimator for tau 